\title{
Perpendicular Magnetization of FePt Alloy Films Epitaxially Grown on Si(100)
}

\author{
Jae Young Ahn, Nyun Jong Lee, and Tae Hee Kim* \\ Department of Physics, Ewha Womans University, Seoul 120-750, Korea
}

(Received 16 November 2009, Received in final form 15 December 2009, Accepted 15 December 2009)

\begin{abstract}
This study examined the structure and perpendicular magnetization of $\mathrm{FePt}$ films grown on $\mathrm{Pt} / \mathrm{Fe} / \mathrm{MgO}(100)$ buffered $\mathrm{Si}(100)$ substrates by molecular beam epitaxy. The $[\mathrm{Fe}(0.17 \mathrm{~nm}) / \mathrm{Pt}(0.2 \mathrm{~nm})]_{\mathrm{N}}$ multilayers were prepared at room temperature to form a $\mathrm{L1}_{0}$-FePt phase after vacuum annealing. Perpendicular magnetic anisotropy (PMA) was observed in the films after at least 15 repetitions $(\mathrm{N}=15)$ of $\mathrm{Fe} / \mathrm{Pt}$ deposition and annealing at $300^{\circ} \mathrm{C}$ for 1 hour. Careful structural analysis of the films was carried out by x-ray diffraction and high-resolution transmission electron microscopy. These results will assist in the development of the low temperature $\mathrm{L1}_{0^{-}}$ FePt deposition process, which will be essential for future extremely high density magnetic recording media.
\end{abstract}

Keywords : perpendicular magnetic anisotropy, $\mathrm{L1}_{0} \mathrm{FePt}$, epitaxial growth, molecular beam epitaxy

\section{Introduction}

The $\mathrm{L} 1_{0}-\mathrm{FePt}$ is the leading candidate for future extremely high density magnetic recording media owing to its very high uniaxial magnetocrystalline anisotropy $\left(\mathrm{K}_{\mathrm{u}}\right)$ $[1,2]$. However, this very high anisotropy imparts high coercivity to these films, which too high for reliable recording using conventional write heads. Moreover, the high temperature process required to form the $\mathrm{L}_{0}$ ordered structure is a disadvantage for practical applications because high temperature durable materials for hard disk drives are yet to be discovered. Some endeavors to develop a low temperature process for fabricating FePt films with a large PMA and reasonable coercivity have been made: in situ annealing [3], use of suitable under layers [4], annealing of multilayers [5], addition of third elements [6], and alternate monatomic layer deposition [7]. Deposition on a heated substrate, which has led to the highest coercivities up to now $[8,9]$, have been explored to achieve chemical ordering. However, the strong in-plane contribution of an easy axis is often observed when annealing films deposited at low temperatures. An earlier report [10] on the important role of strain from a buffer layer on the formation of a $\mathrm{L}_{0}$ phase showed that a highly ordered state can be achieved at low temperatures by exploring an appropriate buffer layer. Moreover, the development of a

*Corresponding author: Tel: +82-2-3277-4647

Fax: +82-2-3277-2372, e-mail: taehee@ewha.ac.kr low temperature fabrication process $\left(\mathrm{T}<400^{\circ} \mathrm{C}\right)$ of highly ordered FePt (001) films, which is ideal for high density perpendicular magnetic recording media applications, is expected considering that the coercivity depends strongly on the thickness and morphology of films [11].

This study examined the low temperature fabrication of $\mathrm{L} 1_{0}$-FePt films grown on $\mathrm{Si}(100)$ substrates using an UHV-molecular beam epitaxy (MBE) thin film deposition technique. To promote $\mathrm{L}_{0}$ ordering in these FePt films, the use of suitable under (or buffer) layers and an alternate monatomic layer deposition technique were evaluated. Control of the magnetic properties and microstructure analysis of the films were attempted systematically considering that the coercivity is a structure sensitive magnetic property.

\section{Experiment}

$[\mathrm{Fe}(\mathrm{x} \mathrm{nm}) / \operatorname{Pt}(0.20 \mathrm{~nm})] \mathrm{x} 15(\mathrm{x}=0.11,0.14$ and 0.17$)$ multilayers were deposited on an epitaxial $\mathrm{Pt} / \mathrm{Fe} / \mathrm{MgO}$ (001) buffer layer grown on chemically etched $\mathrm{Si}(100)$ substrates using an UHV-MBE deposition technique. The base and working pressure was $<2 \times 10^{-10}$ Torr and $<3 \times$ $10^{-9}$ Torr, respectively. The growth temperature of the 5 $\mathrm{nm}$ thick $\mathrm{MgO}$ layer was optimized at $300^{\circ} \mathrm{C}$. A $1 \mathrm{~nm} \mathrm{Fe}$ seed layer and a $10 \mathrm{~nm}$ Pt buffer layer were deposited sequentially at $70^{\circ} \mathrm{C}$. $\sim 5 \mathrm{~nm}$ FePt layers were prepared by the alternate monatomic layered deposition of $\mathrm{Fe}(\mathrm{x} \mathrm{nm})$ and $\mathrm{Pt}(0.2 \mathrm{~nm})$ on a $\mathrm{Pt}$ buffer layer at $70^{\circ} \mathrm{C}$, followed by 
annealing at $300^{\circ} \mathrm{C}$ for 1 hour under vacuum. The typical growth rate was $0.01 \mathrm{~nm} / \mathrm{s}$. A $3 \mathrm{~nm}$ - thick $\mathrm{Al}_{2} \mathrm{O}_{3}$ film, as a capping layer, was deposited on the FePt films at room temperature after annealing.

$\mathrm{X}$-ray powder diffraction (XRD) with $\mathrm{Cu}-\mathrm{K} \alpha$ and transmission electron microscopy (TEM) were used to examine the structure of the films. The magnetic properties were measured using a vibrating sample magnetometer (VSM).

\section{Results and Discussion}

Fig. 1(a) shows XRD patterns of the FePt films grown on the $\mathrm{Pt} / \mathrm{MgO}(001)$ buffered $\mathrm{Si}(100)$ substrates [(a) $\mathrm{x}$ $=0.11$, (b) 0.14 , and (c) $0.17 \mathrm{~nm}]$. The FePt films showed a strong texture in the (001) planes due to the 001 and 002 peaks in the patterns. In addition to the fundamental FePt 002 peak, sample (c) shows more clearly the superlattice FePt 001 peaks associated with the formation of a $\mathrm{L} 1_{0}$ ordered structure. As shown in Fig 1(b), after increasing the Fe monatomic layer thickness slightly from 0.11 to $0.17 \mathrm{~nm}$, i.e., increasing the $\mathrm{Fe}$ content in the system from 41 to 52 at.\%, the superlattice peak positions of the sample (c) were similar to those of the reference-data.

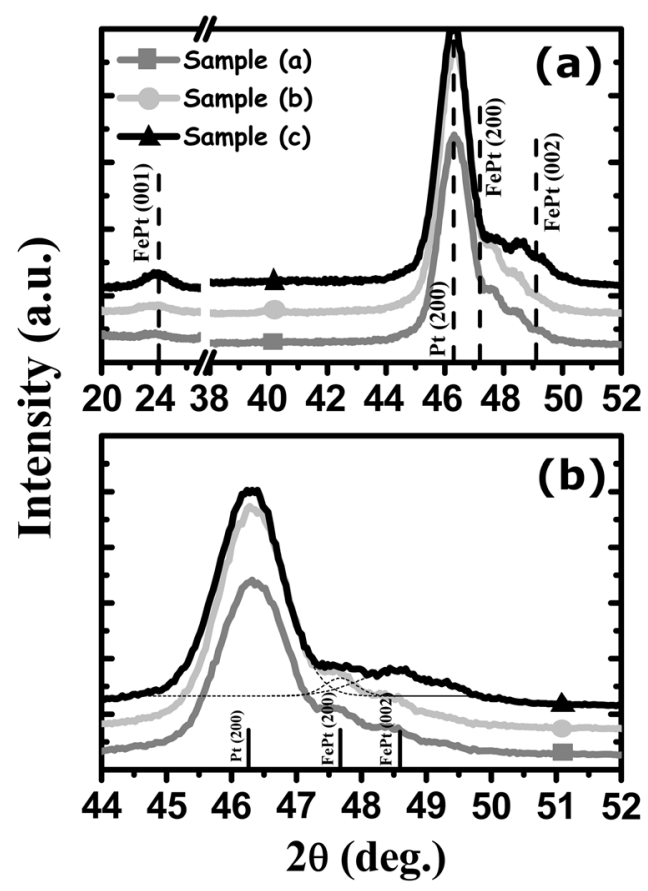

Fig. 1. X-ray diffraction $\theta-2 \theta$ patterns around (a) 20-52 degrees and (b) 42-52 degrees of $[\mathrm{Fe}(\mathrm{x} \mathrm{nm}) / \mathrm{Pt}(0.20 \mathrm{~nm})] \times 15$ films with (a) $\mathrm{x}=0.11$, (b) 0.14 , and (c) $0.17 \mathrm{~nm}$. A $10 \mathrm{~nm} \mathrm{Pt} /$ $1 \mathrm{~nm} \mathrm{Fe} / 5 \mathrm{~nm} \mathrm{MgO}$ multilayer was deposited as a buffer to form the fct-FePt structure; the solid lines represent the FePt peak positions from the powder diffraction file and the dashed lines represent the Gaussian fitting.

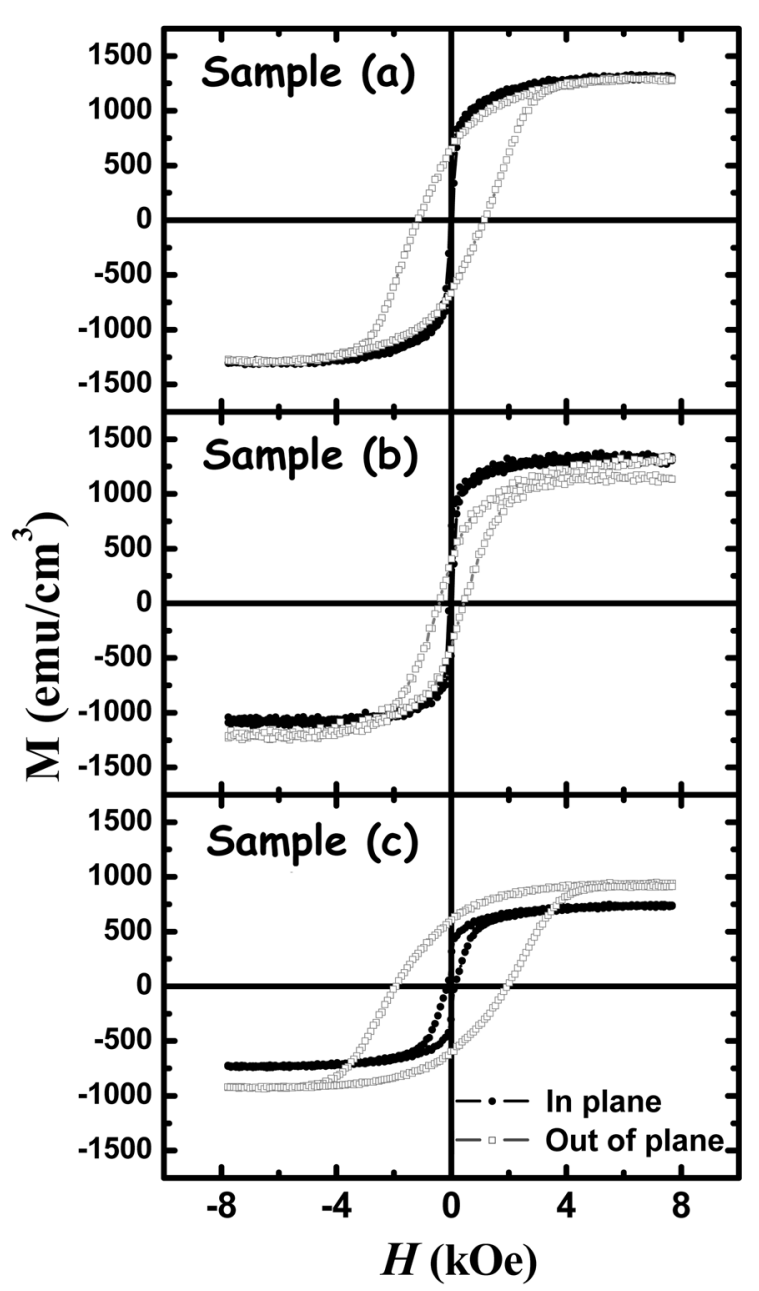

Fig. 2. Magnetization curves measured at room temperature for $[\mathrm{Fe}(\mathrm{x} \mathrm{nm}) / \operatorname{Pt}(0.20 \mathrm{~nm})] \times 15$ films with (a) $\mathrm{x}=0.11$, (b) 0.14 , and (c) $0.17 \mathrm{~nm}$. The magnetic field was applied in the perpendicular direction to the film (open circles) and in the inplane direction (closed circles).

This may be because the structure of sample (c) is similar to the stoichiometric composition, $\mathrm{Fe}_{0.5} \mathrm{Pt}_{0.5}$. These XRD patterns suggest that the structure and texture of the films are sensitive to the Fe composition.

Fig. 2 shows the magnetization curves measured at room temperature for the $[\mathrm{Fe}(\mathrm{x} \mathrm{nm}) / \operatorname{Pt}(0.20 \mathrm{~nm})] \times 15$ films with (a) $x=0.11$, (b) 0.14 , and (c) $0.17 \mathrm{~nm}$. The open and closed circle symbols denote the magnetization curves with the applied fields perpendicular and parallel to the film plane, respectively. The easy magnetization axis is in the perpendicular direction to the film plane for sample (C). This corresponds to the XRD patterns shown in Fig. 1, where the superlattice peak (001) can be observed clearly in the sample (c). An easy axis of the in plane direction to the film plane was achieved in samples (a) and (b), where the $\mathrm{L}_{0}$ ordered structure does not 


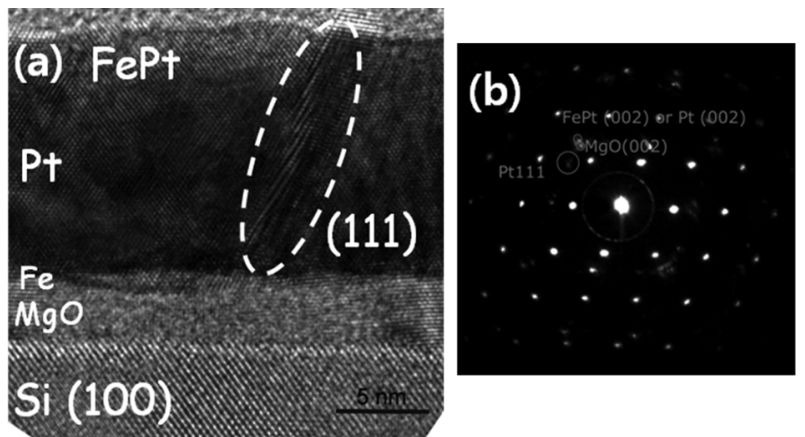

Fig. 3. (a) Cross-sectional TEM image and (b) an electron diffraction pattern for the $[\mathrm{Fe}(0.17 \mathrm{~nm}) / \mathrm{Pt}(0.20 \mathrm{~nm})] \times 15$ film with a $\mathrm{Pt} / \mathrm{Fe} / \mathrm{MgO}$ buffer.

exist. These results show that the easy magnetization axis perpendicular to the film plane results from the formation of a $\mathrm{L}_{0}$ ordered structure with a strong $[00 l]$ texture. However, the chemical ordering in these samples is still far from perfect, showing a high squareness ratio of the M-H loop with the field applied parallel to the film plane. This might be the result of the incomplete formation of the $\mathrm{L} 1_{0}$ phase in these sample due to the low annealing temperature of $300^{\circ} \mathrm{C}$ or insufficient annealing time.

Microstructural analysis using high-resolution-TEM was carried out only on sample (c) because the XRD patterns of samples (a) and (b) indicated poor crystallinity that was unsuitable for TEM analysis. Fig. 3(a) shows crosssection TEM images of sample (c). The results confirmed that the FePt layer grew with a continuous morphology on the $10 \mathrm{~nm}$-thick Pt buffer layer. The epitaxial growth of $\mathrm{MgO}(001)$ on hydrogen terminal $\mathrm{Si}(001)$ with an interface roughness of $9 \AA$ was observed with no visible $\mathrm{SiO}$ oxide layer, only a brighter atomic plane that suggests the presence of light oxygen atoms.

Here, a typical 111-rod shaped grain can be seen in the $\mathrm{Pt}$ and FePt layers, which separates into two 002 oriented grains. The existence of $\mathrm{Pt}$ (111) textured grains was confirmed in the electron diffraction pattern shown in Fig. 3(b). However, excellent crystalline coherency between the Pt buffer and FePt layers was also confirmed, indicating that the FePt layer grew epitaxially on the Pt buffer layer, which is consistent with the result of the magnetic properties of sample (c). The FePt (001) texture mixed with a (111) texture can result in the presence of nonnegligible in-plane easy-axis magnetic anisotropy with predominant perpendicular magnetic anisotropy.

\section{Summary}

This study examined the $\mathrm{Fe}$ composition-dependent magnetic response of FePt films that were grown epitaxially at low temperatures on $\mathrm{Pt} / \mathrm{MgO}(001)$ buffered $\mathrm{Si}(100)$ substrates. Without high temperature annealing ( $\mathrm{T}$ $\left.>400^{\circ} \mathrm{C}\right)$, PMA was observed in the $[\mathrm{Fe}(0.17 \mathrm{~nm}) /$ $\operatorname{Pt}(0.20 \mathrm{~nm})] \times 15$ multilayer film, where the $\mathrm{L} 1_{0}$ ordered structure exists. Microstructural analysis of the film using HR-TEM revealed a FePt $(001)$ texture mixed with a (111) texture. Structural and chemical ordering in the $\mathrm{L} 1_{0} \mathrm{FePt}$ phase is essential for high density perpendicular magnetic media applications. These results suggest that an ideal selective combination of the composition and buffer layer is important for obtaining PMA for $\operatorname{FePt}(001)$ films at reduced temperatures.

\section{Acknowledgment}

This study was supported by Fundamental R\&D Program for Core Technology of Materials funded by the Ministry of Knowledge Economy, Republic of Korea. The structural characterization was performed at the Laboratoire de Physique des Materiaux, Universite de Poitiers, France. The authors wish to thank Professor Anny Michel for her discussion of TEM image analysis.

\section{References}

[1] S. Sun, C. B. Murray, D. Weller, 1. Folks, and A. Moser, Science 287, 1989 (2000).

[2] T. Klemmer et al., Scr. Metall. Mater. 33, 1793 (1995).

[3] Y. K. Takahashi, M. Ohnuma, and K. Hono, Jpn. J. Appl. Phys. 40, L1367 (2001).

[4] Y.-N. Hsu et al., J. Appl. Phys. 89, 7068 (2001).

[5] C. P. Luo and D. J. Sellmyer, IEEE Trans. Magn. 31, 2764 (1995).

[6] T. Maeda, T. Kai, A. Kikisu, T. Nagase, and J. Akiyama, Appl. Phys. Lett. 80, 2147 (2002).

[7] T. Shima, T. Moriguchi, S. Mitani, and K. Takanashi, Appl. Phys. Lett. 80, 288 (2002).

[8] T. Shima, K. Takanashi, Y. K. Takahashi, and K. Hono, Appl. Phys. Lett. 85, 2571 (2004).

[9] M. Weisheit, L. Shultz, and S. Fahler, J. Appl. Phys. 95, 7489 (2004).

[10] T. Seki et al., IEEE Trans. Magn. 40, 2522 (2004).

[11] T. Shima, T. Seki, K. Takanashi, Y. K. Takahashi, and K. Hono, J. Magn. Magn. Mater. 272-276, e557 (2004). 